\title{
BMJ Open Load progression criteria in exercise programmes in lower limb tendinopathy: a systematic review
}

\author{
Adrian Escriche-Escuder (D) , ${ }^{1,2}$ Jose Casaña, ${ }^{3}$ Antonio I Cuesta-Vargas (i) ${ }^{1,2,4}$
}

To cite: Escriche-Escuder A, Casaña J, Cuesta-Vargas Al. Load progression criteria in exercise programmes in lower limb tendinopathy: a systematic review. BMJ Open 2020;10:e041433. doi:10.1136/ bmjopen-2020-041433

- Prepublication history and additional material for this paper is available online. To view these files, please visit the journal online (http://dx.doi.org/10. 1136/bmjopen-2020-041433).

Received 10 June 2020 Revised 01 October 2020 Accepted 05 November 2020

Check for updates

(c) Author(s) (or their employer(s)) 2020. Re-use permitted under CC BY-NC. No commercial re-use. See rights and permissions. Published by BMJ.

${ }^{1}$ Department of Physiotherapy, University of Málaga, Malaga, Spain

${ }^{2}$ Grupo Clinimetría (F-14), Instituto de Investigación Biomédica de Málaga (IBIMA), Malaga, Spain

${ }^{3}$ Department of Physiotherapy, University of Valencia, Valencia, Spain

${ }^{4}$ Health, Queensland University of Technology-QUT, Brisbane, Queensland, Australia

Correspondence to Dr Antonio I Cuesta-Vargas; acuesta@uma.es

\section{ABSTRACT}

Objective The objective of this study is to summarise and analyse the current literature about what progression criteria are applied in loading exercise programmes in lower limb tendinopathies and their evidence and effectiveness.

Design Systematic review.

Methods PubMed, Embase, Scopus and PEDro were searched from inception to 24 September 2020. The inclusion criteria were randomised controlled trials that included patients with midportion Achilles, patellar or gluteal tendinopathy; assessed function, pain or performance; included at least one group where progressive physical exercise was administered as monotherapy; included at least a control group. We excluded studies that included subjects with previous tendon surgical treatment; studies with control group that conducted a supplemented modality of the exercise performed in the intervention group. A narrative synthesis was conducted. Cohen's $d$ and the percentage of change of main clinical and performance outcomes were obtained. Methodological quality was assessed using the PEDro scale.

Results Thirty studies that described progression criteria were included. Six types of criteria grouped in two categories were identified and included in a new classification proposal: pain as a primary criterion (evoking and avoid-pain based), and pain and symptom control as a secondary criterion (conditioning stages, fatigue-based, subjective perception and temporary linear increase). Most of the studies applied a pain-based criterion. Criteria based on conditioning stages were also commonly applied. Other criteria such as fatigue, a temporary linear increase, or the subjective perception of the patient's abilities were occasionally applied.

Conclusions There is a predominant use of pain-based criteria, but the utilisation of these criteria is not supported by strong evidence. This review evidences the need for studies that compare the same exercise programme using different progression criteria. A new classification of the existing progression criteria is proposed based on the use of pain as the primary or secondary criterion.

PROSPERO registration number CRD42018110997.

\section{BACKGROUND}

Progressive therapeutic exercise is considered a first-line treatment in tendinopathies due to the extensive evidence published

\section{Strengths and limitations of this study}

This is the first systematic review that expressly and comprehensively identifies, assesses and summarises the evidence regarding load progression criteria in lower limb tendinopathy.

- This systematic review has been designed and reported according to the Preferred Reporting Items for Systematic Reviews and Meta-Analyses.

- In case of disagreements, a third independent was required for reducing the risk of observer bias.

- A limitation of this systematic review is the noninclusion of studies in which the effect of exercise programmes was studied without a control group, not being included in the analysis and discussion of the results.

- Heterogeneity and deficiencies in the reporting of data found have not allowed the extraction of accurate and conclusive information for developing a quantitative analysis.

in the last three decades. ${ }^{1-5}$ The objective of this treatment modality is to produce mechanical stimulus that provokes biochemical and mechanical responses, generating adaptations of the tendon to load and exercise. ${ }^{1}$ In addition to this, the limited adverse effects produced by therapeutic exercise may explain the growing interest of therapists and patients in this approach. ${ }^{6}$ The current literature shows positive outcomes of exercise programmes on pain and function in different locations of tendinopathies in the upper and lower extremities. ${ }^{3-578}$ Nevertheless, current evidence is not equally consistent for all tendinopathies. In some locations such as the midportion Achilles, patellar or gluteal tendinopathies, the evidence in favour of exercise is abundant, and current studies attempt to elucidate which exercise methodology and dose are most appropriate. ${ }^{4510}$ Meanwhile, the evidence in hamstring, ${ }^{7} 11$ insertional Achilles $^{2}{ }^{12}$ or upper limbs tendinopathies, ${ }^{13}$ among others, is lower, and additional studies supporting exercise programmes are still needed. 
In lower limb tendinopathy, there is not a single modality of therapeutic exercise achieving favourable results, but a broad spectrum of methodologies has been positively applied. Hence, isometric contractions,${ }^{14}$ isolated eccentric training, ${ }^{15}$ combinations of eccentric and concentric contractions ${ }^{16}$ or heavy slow resistance training (HSR $)^{5}$ are some examples of exercise modalities commonly applied in this pathology. Conversely, traditional passive treatments such as corticosteroids injections, ${ }^{35}$ transverse friction ${ }^{17}$ or therapeutic ultrasound ${ }^{17}$ have sometimes not shown enough capacity to maintain positive effects on long-term follow-up.

Cook and Purdam ${ }^{18}$ considered the pathological model of tendinopathy as a continuum, distinguishing three theoretical stages (reactive tendinopathy, tendon disrepair and degenerative tendinopathy). Moreover, a study showed that there is sufficient area with acceptable levels of aligned fibrillar structure in the pathological tendon. ${ }^{19}$ These non-affected areas would be able to compensate for the disorganisation of affected areas by increasing tendon thickness. ${ }^{19}$ According to this approach, the primary stimulus to advance or retreat through the continuum would be adding or removing enough load to obtain changes in the non-affected structure of the tendon. ${ }^{18}$ Thus, it would be necessary individualised handling of the load progression for adequate management of the process. Maximum efficiency is pursued with a reduced risk of injury. For this purpose, some authors have established different methodologies to handle load progression. Nevertheless, there is a lack of consensus and objective criteria on how this load progression should be handled.

In sport and physical training, some authors have suggested that a high risk of injury could not be associated with the use of high loads but with inadequate handling of the progression..$^{20}$ From this approach, the use as a guidance of the $10 \%$ rule among clinicians and trainers is common. According to this rule, it would be essential to control the relationship between the loads applied each week and the average load applied in the previous weeks. Thus, a weekly load progression higher than $10 \%$ would considerably increase the risk of injury. Despite its widespread use, the evidence regarding this rule is controversial. While in some team sports, a significant increase in the risk of injury has been observed with load increases of more than $10 \%$, and especially $50 \%,{ }^{20}$ other studies suggest that in other areas, such as beginning runners, increases between $20 \%$ and $25 \%$ could be well tolerated. ${ }^{21}$ In this context, using the $10 \%$ reasoning only as a guidance seems coherent, if the training experience and the context of each subject for the handling of the load are considered.

In tendinopathies, pain intensity as a load progression criterion is commonly used. Stanish $e t a l^{22}$ and Alfredson et $a l^{15}$ described therapeutic exercise protocols that have been massively used in lower limb tendinopathy. In these protocols, load progression consisted of maintaining a feeling of discomfort or pain during exercises. However, recent systematic reviews have shown that despite its widespread use, there exists a striking lack of evidence for the training parameters applied. ${ }^{9} 23$

Several studies have analysed the effectiveness of different exercise protocols in tendinopathies. ${ }^{45162425}$ Additionally, some of these studies have compared the effect of different symptom management strategies on similar exercise programmes (eg, pain allowed or not allowed during exercise). ${ }^{26} 27$ There exist abundant reviews about pathology, risk factors, prevention, diagnosis or management in lower limb tendinopathies. ${ }^{2}$ However, studies are usually focused on the comparison of different exercise protocols and not on the study of the different progression criteria. Therefore, there is a gap in the evidence on what load progression criterion should be used, which requires an additional analysis of this topic.

As described above, while there is abundant evidence on the effectiveness of exercise in lower limbs tendinopathies, controversy still exists about which may be the best approach in upper limbs. For this reason, this systematic review has only analysed studies concerning tendinopathies of the lower extremities, focusing on the three most predominant ones (Achilles, patellar and gluteal) in order to reduce this heterogeneity. Likewise, those studies concerning insertional Achilles tendinopathy have also been discarded from this review to avoid the heterogeneity caused by its apparent different clinical presentation and response to treatment. ${ }^{2}$

The objective of this systematic review was to summarise and to analyse the current literature on what criteria of progression are applied in loading exercise programmes in lower limb tendinopathies and their evidence and effectiveness.

\section{METHODS}

This systematic review was undertaken following the Preferred Reporting Items for Systematic Reviews and Meta-Analyses guidelines. ${ }^{28}$ Its protocol has been published in an impact journal. ${ }^{29}$

\section{Patient and public involvement}

No patient involved.

\section{Search}

Two reviewers searched PubMed, Embase, Scopus and PED rofrom inception to 24 September 2020. The following search terms relating to the tendinopathy location and exercise were combined for a main search: ("Patellar tendin*" OR "jumper's knee" OR "lander's knee" OR "achilles tendin*" OR "midportion achilles tendin*" OR "mid-portion achilles tendin*" OR "mid-substance Achilles tendin*" OR "midsubstance Achilles tendin*" OR "non-insertional Achilles tendin*" "gluteal tendin*" OR "greater trochanteric bursitis" OR "greater trochanteric pain syndrome" OR "lower limb tendinopathy" OR "tendinopathy" OR "tendonopathy" OR "tendonitis") AND ("exercise" OR "strength" OR "training" OR 
"resistance" OR "loading” OR "progressive” OR "physical activity" OR "eccentric" OR "plyometric" OR "guided imagery" OR "stretching"). Extended information about the searches in the different search engines is provided in online supplemental appendix 1.

\section{Eligibility criteria}

All randomised controlled trials that met the following eligibility criteria based on the PICO framework were included:

1. Participants: people with patellar, midportion Achilles (those studies where the location of the painful area was not specified or where both locations were analysed as a whole were included, considering the predominant incidence of midportion Achilles tendinopathies), or gluteal tendinopathy; at least 16 years old.

2. Interventions: progressive exercise programmes; at least one group where physical exercise was administered as monotherapy. Physical exercise has been defined as a subcategory of physical activity consisting of planned, structured and repetitive movement performed with the purpose of improving or maintaining physical performance or health. ${ }^{30}$ Any voluntary action of the neuromuscular system was considered as physical exercise, including strength training; aerobic exercise; plyometrics; active, self-assisted or guided imagery exercise; active, or self-assisted stretching exercises; other similar forms of exercise; or a combination of these exercises.

3. Control interventions: no intervention, sham or other experimental groups.

4. Outcomes: studies measuring at least function, pain or performance outcomes.

5. No gender, ethnicity, year of publication or language restrictions were imposed.

Those studies that met any of the following exclusion criteria were excluded: (1) including participants with previous tendon surgery; (2) studies in which the exercise was not applied as monotherapy in any of the groups or where the control group involved a supplemented modality of the exercise performed in the intervention group.

\section{Procedures}

All references were imported into the bibliographic management software Mendeley and duplicates were identified and removed. Two independent authors screened the remaining results by title and abstract. Two reviewers screened the full texts of selected articles to identify those that satisfied the eligibility criteria. A third reviewer solved any disagreements.

\section{Data extraction and quality assessment}

Two reviewers retrieved and independently assessed the full texts of the selected studies using an extraction form that included: study setting; study population; participant demographics and baseline characteristics; details of the intervention and control conditions; permission to perform additional physical activity; load progression criteria of the exercise programmes; recruitment and study completion rates; outcomes; effect size (Cohen's d) or percentage of change of a main outcome; significance level; and relevant information about risk of bias. Disagreements between the two reviewers were discussed with a third reviewer. Authors were contacted by email in order to obtain additional information not reported in their articles.

Two reviewers independently assessed the quality and internal validity, as well as the existence of potential bias of the studies using the PEDro scale. ${ }^{31}$ This 11 -item scale is considered a valid and reliable measure of methodological quality of Randomised Controlled Trials (RCTs). ${ }^{31} 32$ Each satisfied item from 2 to 11 (items corresponding to internal validity, that is to say, the extent to which a study establishes a trustworthy cause and effect relationship between a treatment and an outcome) contributes one point to the total PEDro score (0, worst score; 10 , best score). Item 1 pertains to external validity and is not considered for the total score. Therefore, the PEDro scale has the objective of classifying those studies of different methodological quality to allow an adjusted analysis. In this review, those studies scoring 7-10 were considered of good methodological quality, those scoring ranging 5-6 were considered of fair methodological quality, while those that score below 5 were considered of poor quality.

\section{Data synthesis and analysis}

A narrative synthesis to report and compare the different load progression criteria existing in the scientific literature was conducted. Although the authors of most of the studies were contacted by mail in order to obtain the necessary data for inclusion in a meta-analysis, most of these data could not be obtained. Thus, due to the lack of studies with complete data and the existing critical heterogeneity, it was only possible to conduct a narrative synthesis. The different intervention or control groups were organised in the tables by prioritising exercise interventions over passive interventions regardless of the order of interventions in the original studies. In cases where two or more exercise interventions were compared, the intervention that obtained the greatest effect size in the study was prioritised. In all cases, the latest measurement of the main outcome was selected for analysis, thus focusing on the long-term effectiveness of the interventions. Additionally, the heterogeneity of the included studies was assessed ordering the results by methodological characteristics (location of tendinopathy, intervention, type of control group used, progression criteria, data reporting).

The Cohen's $d$ of a main clinical and performance outcome was retrieved or calculated to quantify and compare the effectiveness of the interventions. ${ }^{33}$ Where possible, the Victorian Institute of Sport Assessment (VISA) (VISA-A, VISA-P or VISA-G) questionnaire or Visual Analogue Scale (VAS) was chosen as the main clinical outcome to homogenise the analysis, as they were the most frequently used outcomes. The effect size was 
classified into four levels: $\mathrm{d}<0.2$ was considered a trivial effect size; $d \geq 0.2$ was considered a small effect size; $d \geq 0.5$ was considered a medium effect size, and $d \geq 0.8$ was considered a large effect size. ${ }^{33}$ The significance level was set at 0.05 .

\section{RESULTS}

A total of 9182 citations were identified in PubMed, Embase, Scopus and PEDro with 6870 of them remaining after deleting duplicates. Among these, 117 articles were selected as potentially eligible after reading the title and the abstract (the full text was retrieved in case of doubt).
After evaluating the fulfilment of the eligibility criteria, only 30 studies were included in the systematic review. The flow diagram of the selection process and the reasons for exclusion of the discarded studies are described in figure 1. A complete list of the full-text articles excluded in the last phase is available in online supplemental appendix 2.

\section{Participants}

Online supplemental appendix 3 shows the characteristics of the subjects of the included studies (number of subjects, type of population, age, duration of symptoms

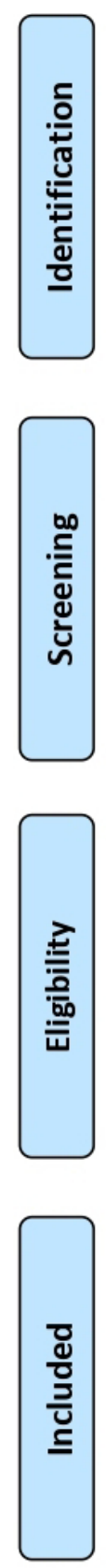

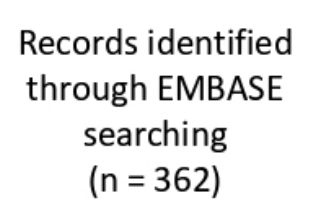

Records identified through EMBASE searching $(n=362)$

$$
\begin{aligned}
& \text { Records identified } \\
& \text { through Pubmed } \\
& \text { searching } \\
& (n=3604)
\end{aligned}
$$

Figure 1 Flow diagram of the selection process.

Studies included in the systematic review $(n=30)$
Records identified through Scopus searching $(n=5019)$

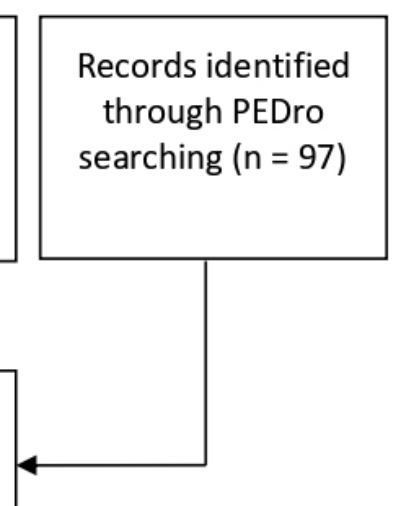

Records excluded $(n=6753)$

Full-text articles excluded, with reasons $(\mathrm{n}=87)$

Exercise versus supplemented exercise $(n=4)$

No exercise $(n=4)$

No exercise as monotherapy $(n=32)$

No progression criteria $(n=7)$

No tendinopathy $(n=4)$

Critical appraisal paper $(n=3)$

Non-randomized study $(n=3)$ No control group $(n=8)$

Study protocol $(n=4)$

A case report $(n=5)$

Case series $(n=2)$

Full-text not available $(n=2)$

Predominant insertional Achilles

tendinopathy $(n=7)$

Age criteria $(n=1)$

Secondary analysis $(n=1)$ 
and information about whether the diagnosis was obtained only clinically or supported by imaging tests).

\section{Exercise programmes}

Different exercise programmes were identified in the included studies: $\mathrm{HSR}^{45}$; isotonic exercise programmes, including both concentric and eccentric phases ${ }^{314} 16$ 34-36; isotonic exercise programmes combined with isometric exercises $^{37}$; isolated isometric exercise programmes ${ }^{143436}$; isolated concentric exercise programmes ${ }^{24} 25839$; eccentric loading programmes ${ }^{4} 16$ 17 24-27 27 37-53 based on the original and modified versions of the Alfredson's protocol ${ }^{15}$; as well as isolated or combined stretching programmes. ${ }^{52} 53$ Online supplemental appendix 4 shows the characteristics of the studies, including the exercise programmes applied in each of them and the permission or not to perform additional physical activity.

\section{Load progression criteria: a proposal for a new classification}

The load progression criteria were identified and included in a new classification proposal. Therefore, the identified criteria were grouped into two categories: pain as a primary progression criterion, and pain and symptom control as a secondary criterion. Two criteria were included in the first category while four criteria were included in the second option of this proposal.

Pain as a primary progression criterion:

1. Evoking pain based (EPB, trying to evoke enough pain to produce improvement): load was gradually increased by using a loaded backpack as pain diminished, aiming at keeping a feeling of pain or discomfort during the exercises. ${ }^{45} 1724262740-4648-54$

2. Avoid pain based (APB, trying to avoid pain): exercises were performed without pain. ${ }^{27} 3738$

Pain and symptom control as a secondary criterion, although pain is controlled and allowed up to a certain limit; progression is marked by other main criteria:

3. Conditioning stages (CS): predefined stages prior to the start of the study, based on the increase in the percentage of the repetition maximum (RM) or on an increase in the complexity of the exercises. ${ }^{3-5} 1624253553$

4. Fatigue based (FB): extra sets or repetitions were performed if there were no signs of fatigue after the first sets. If these are not enough to produce fatigue, weight was gradually increased. ${ }^{47}$

5. Subjective perception (SP): arbitrary increase according to the SP of patient's ability. ${ }^{165-37}$

6. Temporary linear increase (TLI): a linear increase in time (eg, $2.5 \%$ each week). ${ }^{14} 34$

Table 1 shows summary information about the load progression criteria applied in the included studies. Extended information is available in online supplemental appendix 4, along with information about the exercise programmes in which they were included.

\section{Clinical outcomes}

All included studies analysed at least one clinical outcome. The most evaluated outcomes were function using the VISA questionnaire (VISA-A, VISA-P or VISA-G), ${ }^{3-5} 262735-37394042-464849$ and pain using a VAS, ${ }^{451624-2739414749-5155}$ a Numerical Rating Scale, ${ }^{1434363840}$ isolated questions related to pain as painkillers intake, ${ }^{49}$ global rating of change scales, ${ }^{3} 36446$ using dimensions of different questionnaires as the Foot and Ankle Outcome Score questionnaire, ${ }^{54}$ the Hip Dysfunction and Osteoarthritis Outcome Score, the Oxford Hip Score and the Lateral Hip Pain questionnaire, ${ }^{35}$ or self-designed pain scales and questionnaires. ${ }^{53}$ Table 2 shows the Cohen's $\mathrm{d}$, the percentage of change and the significance level (between-group comparison) of a main clinical outcome of each study. Online supplemental appendix 5 provides extended information about all outcomes and measurement timepoints of each study.

\section{Performance outcomes}

In 20 of the 30 studies included in this review, no performance outcomes were evaluated. The most frequently used performance outcomes were the concentric and eccentric torque measured with an isokinetic dynamometer, ${ }^{25} 38475053$ and the jumping performance (countermovement jump test), ${ }^{1624445}$ which were measured in five and four studies, respectively. Other measured performance outcomes were the ankle range of motion ${ }^{16} 42$ or the hip abductor torque. ${ }^{3}$ Table 3 shows the Cohen's $\mathrm{d}$ (between-group comparison), the percentage of change and the significance level of a main performance outcome, where it was possible to obtain or calculate it, of those studies that evaluated at least one performance outcome. Online supplemental appendix 5 provides extended information about the remaining performance outcomes and the measurement timepoints of each study.

\section{Quality assessment}

According to the results of the methodological quality and internal validity analysis (PEDro scale), 12 studies showed a good quality, 14 studies showed a fair quality, while 6 articles were considered of low quality for obtaining a score lower than five points. In general, items 2, 3, 10 and 11 , those involving the random and concealed allocation, the between-group statistical comparisons and the point measures and variability data, showed high compliance (between $83.9 \%$ and $100 \%$ ). Items 4, 7, 8 and 9, including essential items as the similarity of the groups at baseline, the assessor blinding, the number of dropouts and the intention-to-treat analysis showed moderate compliance (between $38.7 \%$ and $64.5 \%$ ). However, items 5 and 6, those that assessed the subject and therapist blinding, showed a negligible compliance rate $(0 \%$ and $3.2 \%$, respectively). Extended information about the compliance of each item is available in table 4 .

\section{DISCUSSION}

A key finding of this systematic review is that load progression is usually influenced by pain perception and symptomatology and not by physical or structural capacity. 
Table 1 Load progression criteria applied in the included studies

\begin{tabular}{lll} 
Study & Cat. & Progression criterion (exercise group 1) \\
\hline Achilles tendinopathy & APB, SP G $^{37}$ & $\begin{array}{l}\text { Patients were briefed to do the exercises } \\
\text { cautiously and pain free, going to the next level } \\
\text { if they were not feeling pain or exhaustion at } \\
\text { maximum load. }\end{array}$
\end{tabular}

\section{Progression criterion 2 (exercise}

Cat. group 2, if any)

\begin{tabular}{|c|c|c|c|c|}
\hline Stefansson et $\mathrm{al}^{42}$ & EPB & $\begin{array}{l}\text { If the patient was pain-free for the full } 15 \\
\text { repetitions for } 3 \text { sets, weight was added for the } \\
\text { next phase. }\end{array}$ & & \\
\hline Beyer et al $\left.\right|^{4}$ & CS & $\begin{array}{l}3 \times 15 \text { repetition maximum ( } 15 \mathrm{RM}) \text {, in week } 1 \text {; } \\
3 \times 12(12 \mathrm{RM}) \text {, in weeks } 2 \text { to } 3 ; 4 \times 10(10 \mathrm{RM}) \text {, in } \\
\text { weeks } 4 \text { to } 5 ; 4 \times 8(8 \mathrm{RM}) \text {, in weeks } 6 \text { to } 8 \text {; and } \\
4 \times 6(6 \mathrm{RM}) \text {, in weeks } 9 \text { to } 12 \text {. }\end{array}$ & EPB & $\begin{array}{l}\text { Load was increased gradually using a } \\
\text { loaded backpack as pain diminished. }\end{array}$ \\
\hline Stevens and $\operatorname{Tan}^{26}$ & EPB & $\begin{array}{l}\text { Load was increased gradually using a loaded } \\
\text { backpack as pain diminished. }\end{array}$ & EPB & $\begin{array}{l}\text { Load was increased gradually using a } \\
\text { loaded backpack as pain diminished. }\end{array}$ \\
\hline
\end{tabular}

Kearney et $a l^{43} \quad$ EPB $\quad$ Progressed as pain allowed. First, by advancing

from double-leg exercises to single-leg

exercises. Second, load was increased gradually using a loaded backpack.

\begin{tabular}{|c|c|c|c|c|}
\hline Yu et $a l^{25}$ & CS & $\begin{array}{l}\text { Different exercises, intensity, and complexity } \\
\text { in each week, according to a stage-based } \\
\text { protocol. }\end{array}$ & CS & $\begin{array}{l}\text { Different exercises, intensity, and } \\
\text { complexity in each week, according to a } \\
\text { stage-based protocol. }\end{array}$ \\
\hline Zhang et $a l^{49}$ & EPB & $\begin{array}{l}\text { Load was increased gradually using a loaded } \\
\text { backpack as pain diminished. }\end{array}$ & & \\
\hline Horstmann et $a l^{47}$ & FB & $\begin{array}{l}\text { Participants performed an extra set if no signs } \\
\text { of fatigue were present after the three first sets. } \\
\text { If necessary, load was increased gradually using } \\
\text { a backpack. }\end{array}$ & & \\
\hline Yelland et $a l^{48}$ & EPB & $\begin{array}{l}\text { Load was increased gradually using a loaded } \\
\text { backpack as pain diminished. }\end{array}$ & & \\
\hline Chester et $a l^{51}$ & EPB & $\begin{array}{l}\text { Load was increased gradually using a loaded } \\
\text { backpack as pain diminished. }\end{array}$ & & \\
\hline Petersen et $a l^{41}$ & EPB & $\begin{array}{l}\text { Load was increased gradually using a loaded } \\
\text { backpack as pain diminished. }\end{array}$ & & \\
\hline Roos et al ${ }^{54}$ & EPB & $\begin{array}{l}\text { Load was increased gradually using a loaded } \\
\text { backpack as pain diminished. }\end{array}$ & & \\
\hline Mafi et $\left.a\right|^{24}$ & EPB & $\begin{array}{l}\text { Load was increased gradually using a loaded } \\
\text { backpack as pain diminished. }\end{array}$ & CS & $\begin{array}{l}\text { Different exercises, intensity, and } \\
\text { complexity in each week, according to a } \\
\text { stage-based protocol. }\end{array}$ \\
\hline Silbernagel et $a l^{16}$ & $\mathrm{CS}, \mathrm{SP}$ & $\begin{array}{l}\text { Different exercises, intensity, and complexity } \\
\text { in each week, according to a stage-based } \\
\text { protocol. Additionally, volume was increased } \\
\text { gradually as ability and symptoms allowed. }\end{array}$ & SP & $\begin{array}{l}\text { Volume and complexity of exercises } \\
\text { were increased gradually as ability and } \\
\text { symptoms allowed. }\end{array}$ \\
\hline
\end{tabular}

APB, SP Patients were briefed to do the exercises cautiously and pain free, going to the next level if they were not feeling pain or exhaustion at maximum feeling loaded backpack as pain diminished. 
Table 1 Continued

\begin{tabular}{lll} 
Study & Cat. & Progression criterion (exercise group 1) \\
\hline Da Cunha et $a l^{27}$ & EPB & $\begin{array}{l}\text { Painful group increased weight to perform } \\
\text { exercise with the greatest pain without altering } \\
\text { performance. }\end{array}$
\end{tabular}

\begin{tabular}{|c|c|c|c|c|}
\hline Kongsgaard et $a l^{5}$ & CS & $\begin{array}{l}4 \times 15 \mathrm{RM}(15 \mathrm{RM}) \text { week } 1 ; 4 \times 12(12 \mathrm{RM}) \text { weeks } \\
2-3 ; 4 \times 10(10 \mathrm{RM}) \text { weeks } 4-5 ; 4 \times 8(8 \mathrm{RM}) \text { weeks } \\
6-8 ; \text { and } 4 \times 6(6 \mathrm{RM}) \text { weeks 9-12. }\end{array}$ & EPB & $\begin{array}{l}\text { Load was increased gradually using a } \\
\text { loaded backpack as pain diminished. }\end{array}$ \\
\hline Frohm et al ${ }^{50}$ & EPB & Increase weight if $\mathrm{VAS}<3$ & EPB & $\begin{array}{l}\text { Increase weight }(5 \mathrm{~kg}) \text { if } \mathrm{VAS}<3 \text {; inertial } \\
\text { exercise: maximal effort. }\end{array}$ \\
\hline Bahr et $a l^{44}$ & EPB & $\begin{array}{l}\text { When pain decreased to }<3 \text {, the participant } \\
\text { added load in a backpack. }\end{array}$ & & \\
\hline Young et $a l^{46}$ & EPB & $\begin{array}{l}\text { Load was increased gradually using a loaded } \\
\text { backpack as pain diminished. }\end{array}$ & EPB & $\begin{array}{l}\text { Progressed as pain diminished (first } \\
\text { from slow to fast, second increasing } \\
\text { load). }\end{array}$ \\
\hline $\begin{array}{l}\text { Jonsson and } \\
\text { Alfredson } \\
39\end{array}$ & EPB & $\begin{array}{l}\text { Load was increased gradually using a loaded } \\
\text { backpack as pain diminished. }\end{array}$ & EPB & $\begin{array}{l}\text { Load was increased gradually using a } \\
\text { loaded backpack as pain diminished. }\end{array}$ \\
\hline \multicolumn{5}{|l|}{ Gluteal tendinopathy } \\
\hline Clifford et $a l^{36}$ & SP & $\begin{array}{l}\text { Exercise progression with the resistance } \\
\text { bands was individualised and based on each } \\
\text { participant's ability to complete the exercises } \\
\text { without increasing their pain beyond } 5 / 10 \text {. }\end{array}$ & SP & $\begin{array}{l}\text { Exercise progression with the resistance } \\
\text { bands was individualised and based on } \\
\text { each participant's ability to complete } \\
\text { the exercises without increasing their } \\
\text { pain beyond } 5 / 10 \text {. All bands }\end{array}$ \\
\hline Mellor et $\left.\mathrm{a}\right|^{3}$ & CS & $\begin{array}{l}\text { Different, exercises, frequency, volume, and } \\
\text { intensity in each week, according to a stage- } \\
\text { based protocol. }\end{array}$ & & \\
\hline
\end{tabular}

.APB, avoiding pain based; Cat., load progression criteria category; CS, conditioning stages; EPB, evoking pain based; FB, fatigue based; SP, subjective perception; TLI, temporary linear increase; VAS, Visual Analogue Scale.

Nevertheless, this fact is not based on an evident demonstration of useful properties of the pain-based criteria, but on a historical inheritance of previous protocols. Although there are a large number of studies focused on comparing different exercise programmes or interventions, this review shows the need for high-quality studies designed to determine the efficacy of a key specific aspect of the programmes such as the load progression criteria. As an additional finding, it has been found that much of the current literature does not provide an appropriate reporting of data (effect size, procedures), which hinders adequate dissemination.
Cat.

Progression criterion 2 (exercise group 2, if any)

APB When the subjects from the 'not painful' group, even without load addition, presented pain during the exercise, they were told to rest the upper limbs on a bar with the purpose to decrease overload on the patellar tendon.

Load was increased gradually using a Increase weight $(5 \mathrm{~kg})$ if $\mathrm{VAS}<3$; inertial exercise: maximal effort.

\section{Achilles and patellar tendinopathies}

Pain-based criteria: evoking and APB

Most of the studies included in this review applied the decrease in discomfort or pain as the primary criterion for increasing the load. This fact has been probably influenced by the large number of studies that investigated the original or a modified version of the isolated eccentric loading programme popularised by Alfredson, ${ }^{15}$ since this is the main criterion used in this procedure. Thus, Alfredson et $a l^{15}$ suggested that the presence of pain is necessary for proper management, ${ }^{15}$ hypothesising that painful eccentric exercises could have a direct mechanical 
Table 2 Cohen's d, percentage of change and significance level (between-group comparison) of main clinical outcomes

\begin{tabular}{|c|c|c|c|c|c|}
\hline \multirow{2}{*}{ Study } & \multicolumn{5}{|l|}{ Results } \\
\hline & Clinical outcome & Time & Cohen's d (main outcome) & $\%$ of change & $P$ value \\
\hline \multicolumn{6}{|l|}{ Achilles tendinopathy } \\
\hline Stefansson et $a l^{42}$ & VISA-A-IS & 24 weeks & N/A & $\begin{array}{l}\text { EPB (ECC) - Pressure } \\
\text { massage: N/A }\end{array}$ & $\begin{array}{l}\text { Pressure massage } \\
\text {-EPB (ECC): }>0.05\end{array}$ \\
\hline Beyer et $a l^{4}$ & VISA-A & 52 weeks & $\begin{array}{l}\text { CS (HSR) - EPB (ECC): } \\
1.66\end{array}$ & $\begin{array}{l}62.96 \% \text { CS (HSR) - } \\
46.55 \% \text { EPB (ECC) }\end{array}$ & HSR - ECC: $>0.05$ \\
\hline Stevens and $\operatorname{Tan}^{26}$ & VISA-A & 6 weeks & $\begin{array}{l}\text { (EPB) Do as tolerated ECC } \\
\text { - Standard ECC } 0.42\end{array}$ & $\begin{array}{l}32.69 \% \text { Do as tolerated - } \\
18.34 \% \text { Standard }\end{array}$ & $>0.05$ \\
\hline Kearney et $\left.a\right|^{43}$ & VISA-A & 26 weeks & EPB (ECC) - PRP: -0.55 & $\begin{array}{l}58.33 \% \text { ECC - } \\
85.36 \% \text { PRP }\end{array}$ & $>0.05$ \\
\hline Horstmann et $a l^{47}$ & $\begin{array}{l}\text { VAS: palpation pain } \\
2 \mathrm{~cm} \text { proximal to } \\
\text { insertion }\end{array}$ & 12 weeks & $\begin{array}{l}\text { FB (ECC) - Wait and see: } \\
0.89 ; \text { FB (ECC) - Whole- } \\
\text { body vibration: } 0.27\end{array}$ & $\begin{array}{l}\text { 67.24\% FB (ECC); } \\
51.44 \% \text { Vibration; } \\
\quad 27.95 \% \text { Wait and see }\end{array}$ & $<0.05^{*}$ \\
\hline Yelland et $a l^{48}$ & VISA-A & 52 weeks & $\begin{array}{l}\text { EPB (ECC) - Prolotherapy } \\
\text { injections: }-0.09\end{array}$ & $\mathrm{~N} / \mathrm{A}$ & $>0.05$ \\
\hline Chester et $a l^{51}$ & VAS rest & 12 weeks & $\begin{array}{l}\text { EPB (ECC) - Therapeutic } \\
\text { ultrasound: }-0.05\end{array}$ & $\begin{array}{l}4.00 \% \text { EPB (ECC) - } \\
7.01 \% \text { Therapeutic } \\
\text { ultrasound: }\end{array}$ & $>0.05$ \\
\hline Petersen et al ${ }^{41}$ & $\begin{array}{l}\text { VAS daily living } \\
\text { activities }\end{array}$ & 54 weeks & $\mathrm{N} / \mathrm{A}$ & $\begin{array}{l}30 \% \text { EPB (ECC) - } 27 \% \\
\text { Brace }\end{array}$ & $<0.05^{\star}$ \\
\hline Mafi et $a l^{24}$ & $\begin{array}{l}\text { VAS during activity } \\
\text { (running or walking) }\end{array}$ & 12 weeks & $\mathrm{N} / \mathrm{A}$ & $\begin{array}{l}\text { EPB (ECC) - CS (CONC): } \\
\text { N/A }\end{array}$ & N/A \\
\hline Silbernagel et $\mathrm{al}^{16}$ & VAS on palpation & 26 weeks & $\begin{array}{l}\text { CS, SP }(E C C+C O N C)-S P \\
(E C C): 0.42\end{array}$ & $\begin{aligned} & 57.14 \% \mathrm{CS}, \mathrm{SP} \\
& (\mathrm{ECC}+\mathrm{CONC})- \\
& 66.67 \% \mathrm{SP}(\mathrm{ECC})\end{aligned}$ & $>0.05$ \\
\hline $\begin{array}{l}\text { Niesen-Vertommen } \\
\text { et } a /^{67}\end{array}$ & $\begin{array}{l}\text { Numerical Rating } \\
\text { Scale }\end{array}$ & 12 weeks & $\mathrm{N} / \mathrm{A}$ & $\begin{array}{l}78.33 \% \text { APB (ECC) - } \\
-46.15 \% \text { APB (CONC) }\end{array}$ & $\mathrm{N} / \mathrm{A}$ \\
\hline \multicolumn{6}{|l|}{ Patellar tendinopathy } \\
\hline Rio et $a l^{14}$ & $\begin{array}{l}\text { Pain measured } \\
\text { with a Numerical } \\
\text { Rating Scale during } \\
\text { a single leg decline } \\
\text { squat }\end{array}$ & 4 weeks & $\begin{array}{l}\text { TLI (ISOM) - TLI (Isotonic): } \\
2.75\end{array}$ & $\mathrm{~N} / \mathrm{A}$ & $<0.05^{\star}$ \\
\hline van Ark et al ${ }^{34}$ & $\begin{array}{l}\text { Pain measured } \\
\text { with a Numerical } \\
\text { Rating Scale during } \\
\text { a single leg decline } \\
\text { squat }\end{array}$ & 4 weeks & N/A & $\begin{array}{l}\text { 63,63\% TLI (Isotonic) - } \\
36,50 \% \text { TLI (ISOM) }\end{array}$ & $>0.05$ \\
\hline Da Cunha et $a l^{27}$ & VISA-P & 12 weeks & $\mathrm{N} / \mathrm{A}$ & $\begin{array}{l}\text { EPB (Decline Board ECC) } \\
\text { - APB (Decline Board ECC) } \\
\text { N/A }\end{array}$ & $>0.05$ \\
\hline
\end{tabular}


Table 2 Continued

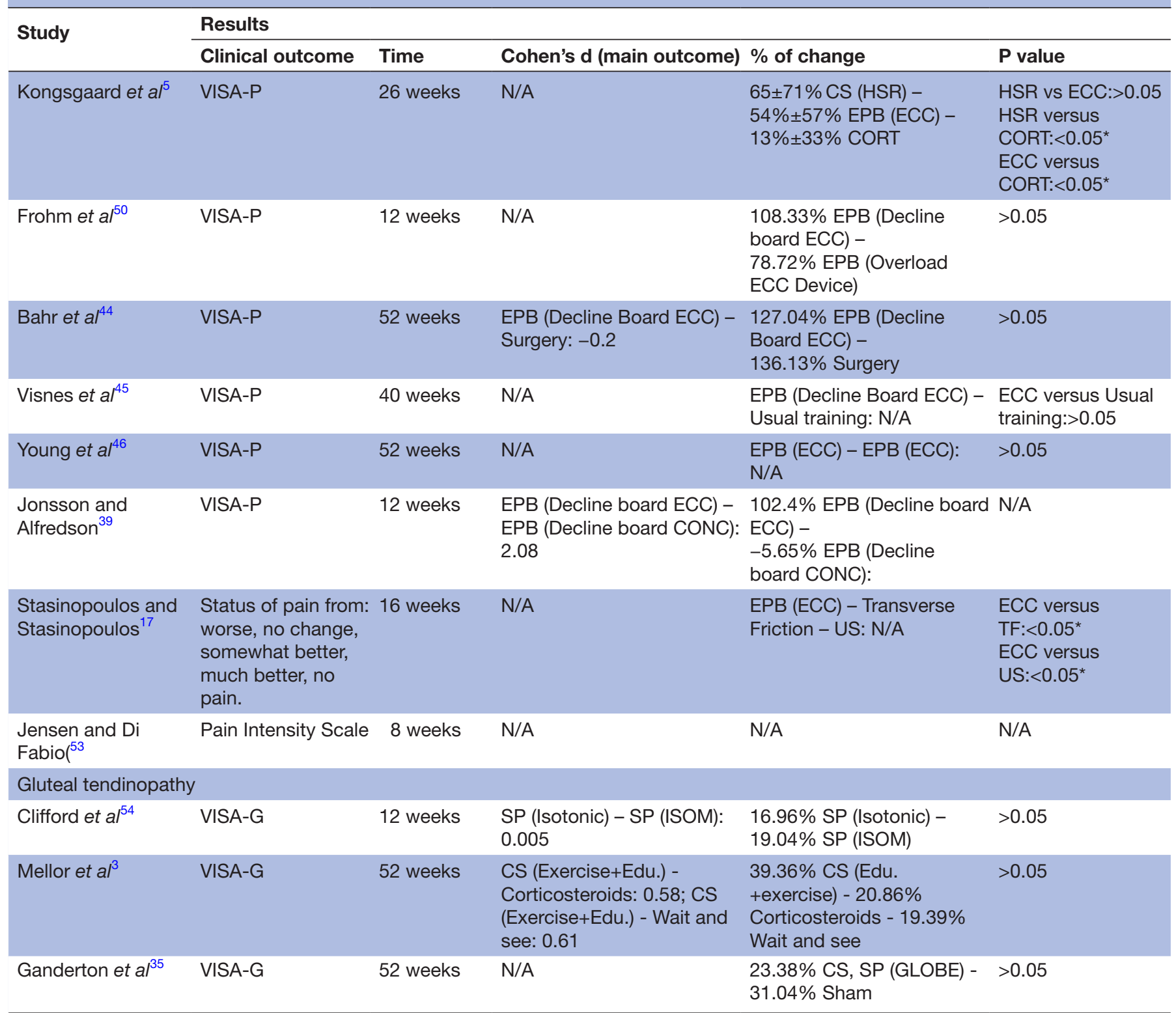

*Significant differences between groups.

APB, avoiding pain-based; CONC, isolated concentric exercise; CORT, corticosteroid injections; CS, conditioning stages; ECC, isolated eccentric exercise; Edu, education; EPB, evoking pain-based; FAOS, Foot and Ankle Outcome Score; FB, fatigue-based; GLOBE, Gluteal La Trobe University exercise program; HSR, heavy slow resistance training; ISOM, Isometrics; N/A, not available; p, significance level; PRP, platelet rich plasma; SP, subjective perception; SWT, shockwave therapy; TF, transverse friction; TLI, temporary linear increase; US, ultrasound therapy; VAS, Visual Analogue Scale; VISA-A, Victorian Institute of Sport Assessment Questionnaire for Achilles tendon; VISA-G, Victorian Institute of Sport Assessment Questionnaire for gluteal tendinopathy; VISA-P, Victorian Institute of Sport Assessment Questionnaire for patellar tendon.

effect on neurovascular ingrowth that may be a source of symptoms. ${ }^{56}$

In this review, the results obtained by the studies that applied an EPB criterion in Achilles and patellar tendinopathies were similar. Maintaining a constant feeling of pain or discomfort according to the description of 'load was increased gradually using a backpack (or weights in hands) as pain diminished' was the most frequently used criterion. This specific criterion was used in 20 of the 30 studies, most of them applying isolated eccentric exercise programmes. The combination of this EPB criterion with isolated eccentric training only achieved favourable significant differences in the VISA-A questionnaire versus a non-intervention group in Achilles ${ }^{40}$ and versus ultrasound therapy and transverse friction massage in patellar tendinopathy. ${ }^{17}$ Nevertheless, a passive therapy such as acupuncture was found to be significantly better than this approach. In both locations, the combination of this progression criterion with the isolated eccentric training did not show significant differences in VISA-A 


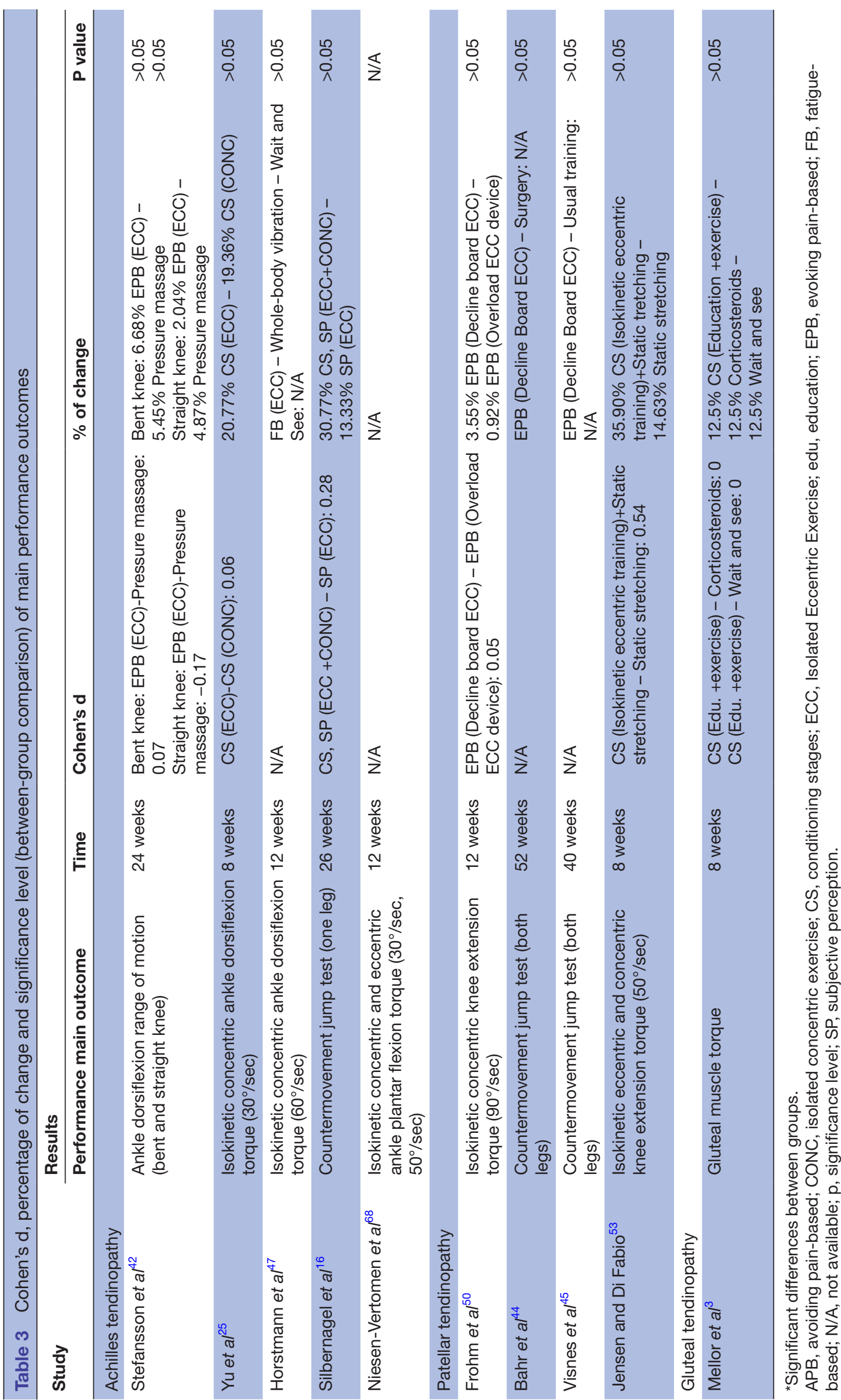


Table 4 Internal validity analysis (PEDro scale)

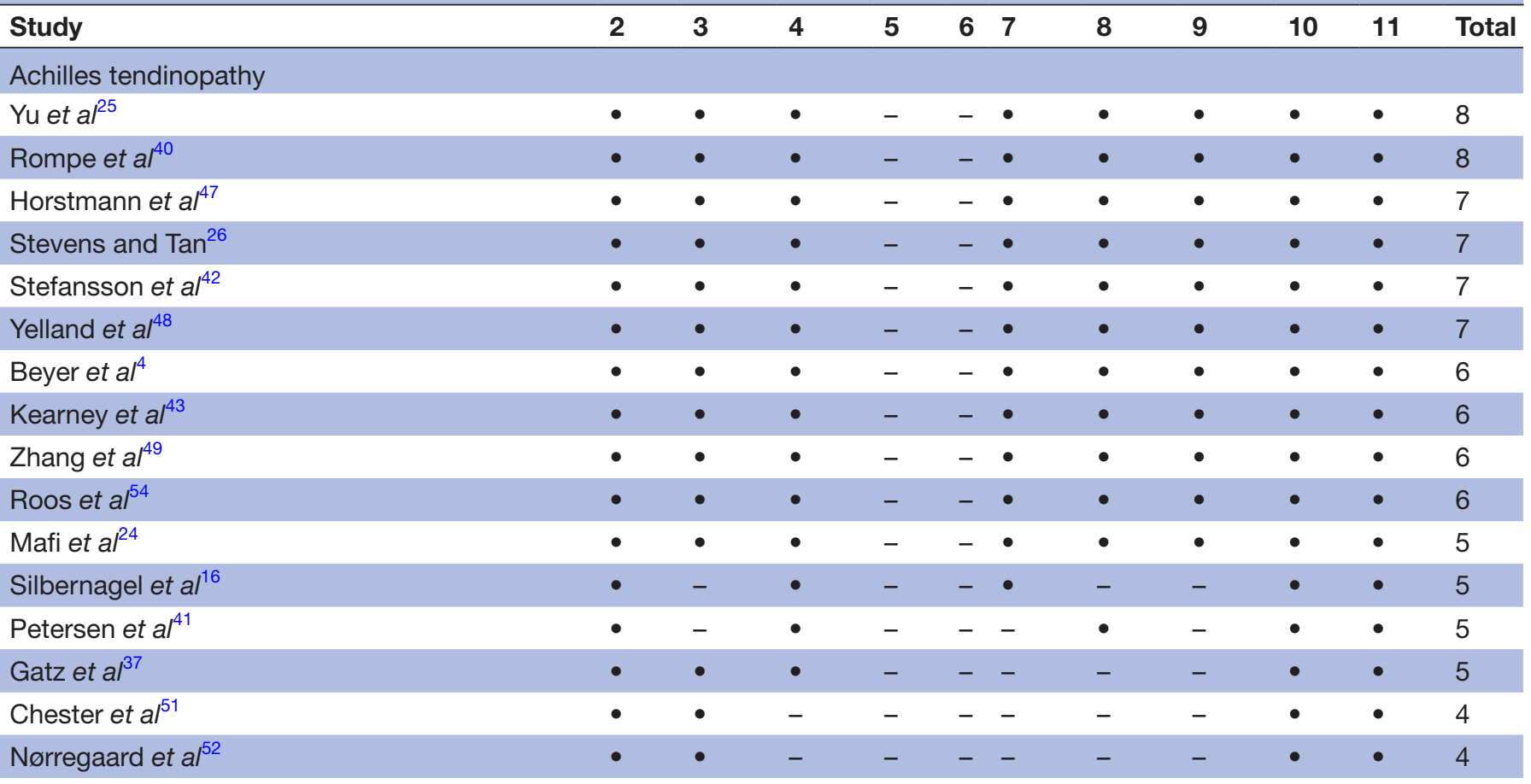

Patellar tendinopathy

\begin{tabular}{|c|c|c|c|c|c|c|c|c|c|c|c|}
\hline Kongsgaard et $a l^{5}$ & $\bullet$ & $\bullet$ & $\bullet$ & - & - & - & $\bullet$ & - & $\bullet$ & $\bullet$ & 7 \\
\hline Bahr et $a l^{44}$ & $\bullet$ & $\bullet$ & $\bullet$ & - & - & - & $\bullet$ & $\bullet$ & $\bullet$ & $\bullet$ & 7 \\
\hline Stasinopoulos and Stasinopoulos ${ }^{17}$ & $\bullet$ & $\bullet$ & - & - & - & - & $\bullet$ & $\bullet$ & $\bullet$ & - & 7 \\
\hline Rio et $a l^{14}$ & $\bullet$ & $\bullet$ & $\bullet$ & - & - & - & - & $\bullet$ & $\bullet$ & $\bullet$ & 6 \\
\hline Frohm et $a l^{50}$ & $\bullet$ & $\bullet$ & $\bullet$ & - & - & - & $\bullet$ & - & $\bullet$ & $\bullet$ & 6 \\
\hline van Ark et $a l^{34}$ & $\bullet$ & $\bullet$ & $\bullet$ & - & - & - & - & - & $\bullet$ & $\bullet$ & 5 \\
\hline Jensen and Di Fabio ${ }^{53}$ & $\bullet$ & - & - & - & - & - & $\bullet$ & - & $\bullet$ & $\bullet$ & 4 \\
\hline Jonsson and Alfredson ${ }^{39}$ & $\bullet$ & - & - & - & - & - & - & - & $\bullet$ & $\bullet$ & 3 \\
\hline \multicolumn{12}{|l|}{ Gluteal tendinopathy } \\
\hline$\%$ Of agreement & 100 & 83.9 & 64.5 & 3.2 & 0 & 38.7 & 61.3 & 41.9 & 100 & 100 & \\
\hline
\end{tabular}

-Yes; -: no. 2: Random allocation; 3: Concealed allocation; 4: Groups similar at baseline; 5: Subject blinding; 6: Therapist blinding; 7: Assessor blinding; 8: Less than 15\% dropouts; 9: Intention-to-treat analysis; 10: Between-group statistical comparisons; 11: Point measures and variability data.

or VISA-P versus an programme based on $\mathrm{CS} .{ }^{45}$ In terms of pain assessment, although significant differences were observed in favour of the group with the EPB criterion versus the placement of a brace, ${ }^{41}$ the results contrast with another study that found no differences versus the placement of a night splint. ${ }^{54}$

Five more studies ${ }^{42-46}$ applied this combination of the EPB criterion and eccentrics. However, the progression was implemented differently. Although in all cases differences within the group were obtained, none of these studies obtained significant differences in the comparison between groups.

Three studies described an avoiding pain-based progression criterion. da Cunha et $a l^{27}$ compared the effectiveness of two isolated eccentric programmes, performing the exercise with the greatest pain without altering performance and with the avoiding pain-based criterion, respectively, not showing significant differences in 
VISA-P between groups. These results could be related with the findings of the study of Stevens and Tan, ${ }^{26}$ where performing the Alfredson's protocol following instructions of 'do the protocol as tolerated' achieved better short-term (6 weeks) results in VISA-A than the standard protocol (although without significant differences). Gatz $e t a \hat{l}^{37}$ and Niesen-Vertommen et $a l^{38}$ assessed the effectiveness of an isolated eccentric protocol versus a combination of isometric and eccentric exercises and versus a concentric exercise programme, respectively. In both studies, the progression was based on the absence of discomfort in the last serie. None of the two studies showed differences between groups, all of them using the same progression criterion.

Although the heterogeneity of the studies included in this review does not allow for robust conclusions, these findings do not support the need to apply EPB progression criteria as a primary option. So although monitoring pain could be important and some studies have previously related changes in rating pain scales to their clinical importance,${ }^{57}$ it could not be the most appropriate criterion to establish load progression in therapeutic exercise programmes. Thus, the use of a pain-based criterion instead of an individualised criterion for neuromuscular capacity and function could overestimate or underestimate the actual capacity of the system. Therefore, there is still a gap in the existing knowledge about the relation between pain-based criteria and the optimal load in exercise programmes.

\section{Conditioning stages}

As an alternative option to a primary pain-based progression criterion, other procedures have been described based on individualised aspects of the patients, such as the load the subjects could handle each week or their current abilities. The use of predefined $C S$ in which each week or group of weeks had a previously determined work intensity, usually based on a percentage of the RM, but also on current abilities of the patient, has also been a commonly applied criterion among the included studies. This criterion, commonly used in sports and physical training, has been included in the last decades in numerous programmes of clinical exercise, also showing beneficial effects. ${ }^{58}$ This step-based approach using a progression in the percentage of the maximum repetition ensures a progression in intensity while allowing individualisation of the load based on the patient's current capacity. In this review, most of the included studies that have applied this criterion have done so by comparing it to other exercise groups that also used stage-based criteria, ${ }^{16}{ }^{25}$ which makes it difficult to draw conclusions. As described above, Beyer $e t a t^{4}$ and Kongsgaard $e t a \tilde{e}$ in Achilles and patellar tendinopathy, respectively, found a larger effect size in the HSR group that applied this criterion than in the isolated eccentric training group that used a pain-based criterion, although these differences were not statistically significant. Additionally, in one of these studies, the good clinical effects observed in the HSR group were accompanied by reductions of tendon abnormality and an increased collagen turnover not found with the EPB criterion of the isolated eccentric group. ${ }^{5}$ However, the existing evidence is still not enough to determine that this criterion is the most appropriate.

Although no similar studies have been found in the study population, a previous study carried out in plantar fasciopathy did not find any differences in pain reduction between performing an HSR protocol based on predetermined stages compared and a group that performed the same exercises in a self-administered manner, allowing work with the highest tolerated load from the start, setting the load margins of the group based on stages as limits. ${ }^{59}$ These findings may suggest that the effectiveness of the CS criteria may be related to the individualised calculation of the percentage of the RM and the observation of the current capacities of the patients, and not to the division into stages of the programme.

\section{TLI, FB and SP criteria}

To a lesser extent, other criteria applied in the included studies were the use of a TLI (eg, $2.5 \%$ weekly) where possible, fatigue control, or an arbitrary increase in volume (series or repetitions) or intensity where it was considered subjectively necessary.

Two studies increased weight by $2.5 \%$ every week where possible as a progression criterion, including isolated isometric and isotonic exercise programmes. ${ }^{14} 34$ Additional studies comparing this criterion to others are still necessary. However, it seems evident that the use of these linear criteria does not allow load individualisation, since the increase of an absolute percentage $(2.5 \%$ in the example) can mean very different variations in individuals with different capacities, which may reduce the potential effectiveness of the programmes where it is applied.

Only one study applied a FB criterion. ${ }^{47}$ In this study, significant differences were found in pain on palpation (measured with VAS) in favour of the exercise group versus a whole body vibration and a non-intervention group. Nevertheless, no significant differences were found in the isokinetic concentric ankle dorsiflexion torque $\left(60^{\circ} / \mathrm{sec}\right)$ performance outcome.

Two studies considered the SP of the current participant's abilities and skills as the main progression criterion. ${ }^{16}{ }^{36}$ In Silbernagel $e t a l,{ }^{16}$ this criterion was applied in combination with a CS criterion. Thus, although the progression over the weeks was previously predefined, the progression was supervised by a physiotherapist and dependent on the patient's ability and symptoms. This study did not find significant differences in the pain or performance outcomes of the addition of a SP to a CS criterion versus the isolated CS criterion. ${ }^{16}$

\section{Gluteal tendinopathy}

Regarding gluteal tendinopathy, only three studies were included in this review. Mellor $e t a \hat{l}$ and Ganderton $e t a l^{55}$ compared the effectiveness of an exercise and education programme, finding no significant differences in the 
VISA-G versus any of the control groups. Both studies applied a CS criterion. Moreover, in one of the studies, ${ }^{35}$ the progression through the stages was additionally dependent on the patient's abilities. Clifford $e t a l^{36}$ did not find significant differences in VISA-G between a group performing an isometric isolated eccentric programme versus a group applying a isotonic exercise programme, both with a progression criteria based on the participant's ability to complete the exercises. Clifford $e t a l^{36}$ did not find significant differences in VISA-G between a group performing an isometric isolated eccentric programme versus a group applying a isotonic exercise programme, both with a progression criteria based on the participant's ability to complete the exercises.

\section{Study outcomes}

The widespread use of the VISA questionnaire (in its different versions) and the VAS scale for pain has allowed some degree of homogeneity in the clinical outcomes studied in the current literature. However, an additional finding of this review is that despite the growing knowledge about the importance of performance outcomes in tendinopathy and the controversial relationship of pain and structure with function and recovery of the tendon, no performance outcomes were measured in most of the studies included in this review.

\section{Additional physical activity}

The fact that the studies were not homogeneous in the prohibition of performing additional physical activity during the programme may have influenced the results of the different protocols and criteria. Nevertheless, a previous systematic review showed that there is no strong evidence supporting the need of withdrawal from the sport in the management of patellar tendinopathy, ${ }^{60}$ so the possible influence of the additional activity must still be verified.

\section{What has been excluded from this systematic review?}

Due to the selection criteria chosen for this review, several studies have not been included, as they compare exercise interventions versus supplemented exercise. Although this is a common practice in the research of complementary therapies, it does not allow a proper analysis of the programmes. ${ }^{61-63}$ During the selection phase, a significant number of studies including exercise with no load progression were identified, but they were excluded from the review. A lack of analysis of structural outcomes such us thickness has been found. This may be due to the fact that the studies where structural variables are analysed are usually designed as non-controlled longitudinal prospective studies using MRI. ${ }^{64-66}$ Finally, describing well-designed high-quality study protocols have been found but they are not available yet. ${ }^{1067}$

\section{Strengths and limitations}

The main strength of this study is that has identified a significant gap in the literature that future studies should fill. Another essential strength is the proposal for a new approach presented for the study of exercise programmes in tendinopathies, based on a possible new classification of the different progression criteria in loading exercise. However, heterogeneity and deficiencies in the reporting of data found have not allowed the extraction of accurate and conclusive information, not allowing to fulfil the second of the purposes set in this review: the study of the efectiveness. Some limitations are the absence of washout from previous treatments in most of the studies, and the permission to take analgesics or non-steroidal anti-inflammatory drugs in some studies. Additionally, there is a potential selection and reporting bias from those studies that may only be present in databases that have not been consulted. These bias have been tried to reduce by increasing the number of databases analysed and expanding the selection criteria.

Future studies comparing interventions applying different load progression criteria to the same exercise programme are needed, allowing a trustworthy review of the subject. In addition, it is necessary to search for new progression criteria adapted to the existing knowledge, as well as for more accurate information about neuromuscular ability, training parameters, the minimum number of sessions required, or the adherence levels of exercise programmes.

\section{CONCLUSIONS}

Despite the limitations, this systematic review offers a comprehensive summary of the current evidence regarding the load progression criteria in lower limb tendinopathy.

The findings of this systematic review reveal a predominant use of pain-based criteria, which is the result of a historical and scientific inheritance of exercise protocols but it is not supported by strong evidence. The lack of evidence found regarding the effectiveness of the commonly applied load progression criteria and the contradictory results of existing studies make it essential to study and search for new criteria that can be supported by the current knowledge and evidence. Thus, the current criteria should be used by practitioners cautiously and critically, waiting for strong evidence to support their use.

Contributors All authors contributed to the study design. AE-E and AIC-V searched and screened the articles, with assistance from JC. All authors contributed to data analysis and interpretation of the data. AE-E drafted the manuscript, AIC-V and JC revised it critically, and all authors contributed to revisions and approved the final manuscript.

Funding This work is part of a government-funded project supported by the University Teaching Training Programme (FPU) of the Ministry of Science, Innovation and Universities of Spain. Grant number: FPU17/00161. The University of Malaga has supported this study with the contribution of funds to support its publication in open access.

Competing interests None declared.

Patient consent for publication Not required.

Provenance and peer review Not commissioned; externally peer reviewed.

Data availability statement № additional data available. 
Supplemental material This content has been supplied by the author(s). It has not been vetted by BMJ Publishing Group Limited (BMJ) and may not have been peer-reviewed. Any opinions or recommendations discussed are solely those of the author(s) and are not endorsed by BMJ. BMJ disclaims all liability and responsibility arising from any reliance placed on the content. Where the content includes any translated material, BMJ does not warrant the accuracy and reliability of the translations (including but not limited to local regulations, clinical guidelines, terminology, drug names and drug dosages), and is not responsible for any error and/or omissions arising from translation and adaptation or otherwise.

Open access This is an open access article distributed in accordance with the Creative Commons Attribution Non Commercial (CC BY-NC 4.0) license, which permits others to distribute, remix, adapt, build upon this work non-commercially, and license their derivative works on different terms, provided the original work is properly cited, appropriate credit is given, any changes made indicated, and the use is non-commercial. See: http://creativecommons.org/licenses/by-nc/4.0/.

\section{ORCID iDs}

Adrian Escriche-Escuder http://orcid.org/0000-0003-4402-6483

Antonio I Cuesta-Vargas http://orcid.org/0000-0002-8880-4315

\section{REFERENCES}

1 Docking SI, Cook J. How do tendons adapt? going beyond tissue responses to understand positive adaptation and pathology development: a narrative review. J Musculoskelet Neuronal Interact 2019;19:300-10.

2 Cardoso TB, Pizzari T, Kinsella R, et al. Current trends in tendinopathy management. Best Pract Res Clin Rheumatol 2019;33:122-40.

3 Mellor R, Bennell K, Grimaldi A, et al. Education plus exercise versus corticosteroid injection use versus a wait and see approach on global outcome and pain from gluteal tendinopathy: prospective, single blinded, randomised clinical trial. BMJ 2018;361:k1662.

4 Beyer R, Kongsgaard M, Hougs Kjær B, et al. Heavy slow resistance versus eccentric training as treatment for Achilles tendinopathy: a randomized controlled trial. Am J Sports Med 2015;43:1704-11.

5 Kongsgaard M, Kovanen V, Aagaard P, et al. Corticosteroid injections, eccentric decline squat training and heavy slow resistance training in Patellar tendinopathy. Scand J Med Sci Sports 2009;19:790-802.

6 Niemeijer A, Lund $\mathrm{H}$, Stafne SN, et al. Adverse events of exercise therapy in randomised controlled trials: a systematic review and meta-analysis. Br J Sports Med 2019:bjsports-2018-100461.

7 Cushman D, Rho ME. Conservative treatment of subacute proximal hamstring tendinopathy using eccentric exercises performed with a treadmill: a case report. J Orthop Sports Phys Ther 2015;45:557-62.

8 Pienimäki TT, Tarvainen TK, Siira PT, et al. Progressive strengthening and stretching exercises and ultrasound for chronic lateral epicondylitis. Physiotherapy 1996;82:522-30.

9 Habets B, van Cingel REH. Eccentric exercise training in chronic mid-portion Achilles tendinopathy: a systematic review on different protocols. Scand J Med Sci Sports 2015;25:3-15

10 Habets B, van Cingel REH, Backx FJG, et al. Alfredson versus Silbernagel exercise therapy in chronic midportion Achilles tendinopathy: study protocol for a randomized controlled trial. BMC Musculoskelet Disord 2017;18:296.

11 Chu SK, Rho ME. Hamstring injuries in the athlete: diagnosis, treatment, and return to play. Curr Sports Med Rep 2016;15:184-90.

12 Rompe JD, Furia J, Maffulli N. Eccentric loading compared with shock wave treatment for chronic insertional Achilles tendinopathy. A randomized, controlled trial. J Bone Joint Surg Am 2008;90:52-61.

13 Ortega-Castillo M, Medina-Porqueres I. Effectiveness of the eccentric exercise therapy in physically active adults with symptomatic shoulder impingement or lateral epicondylar tendinopathy: a systematic review. J Sci Med Sport 2016;19:438-53.

14 Rio E, van Ark M, Docking S, et al. Isometric contractions are more analgesic than isotonic contractions for Patellar tendon pain: an InSeason randomized clinical trial. Clin J Sport Med 2017;27:253-9.

15 Alfredson H, Pietilä T, Jonsson P, et al. Heavy-load eccentric calf muscle training for the treatment of chronic Achilles tendinosis. Am J Sports Med 1998;26:360-6.

16 Silbernagel KG, Thomeé R, Thomeé P, et al. Eccentric overload training for patients with chronic Achilles tendon pain--a randomised controlled study with reliability testing of the evaluation methods. Scand J Med Sci Sports 2001;11:197-206.

17 Stasinopoulos D, Stasinopoulos I. Comparison of effects of exercise programme, pulsed ultrasound and transverse friction in the treatment of chronic Patellar tendinopathy. Clin Rehabil 2004;18:347-52.

18 Cook JL, Purdam CR. Is tendon pathology a continuum? A pathology model to explain the clinical presentation of load-induced tendinopathy. Br J Sports Med 2009;43:409-16.

19 Docking SI, Cook J. Pathological tendons maintain sufficient aligned fibrillar structure on ultrasound tissue characterization (UTC). Scand J Med Sci Sports 2016;26:675-83.

20 Gabbett TJ. The training-injury prevention paradox: should athletes be training smarter and harder? Br J Sports Med 2016;50:273-80.

21 Nielsen RO, Cederholm P, Buist I, et al. Can GPs be used to detect deleterious progression in training volume among runners? $J$ Strength Cond Res 2013;27:1471-8.

22 Stanish WD, Rubinovich RM, Curwin S. Eccentric exercise in chronic tendinitis. Clin Orthop Relat Res 1986:65-8.

23 Malliaras P, Barton CJ, Reeves ND, et al. Achilles and patellar tendinopathy loading programmes : a systematic review comparing clinical outcomes and identifying potential mechanisms for effectiveness. Sports Med 2013;43:267-86.

24 Mafi N, Lorentzon R, Alfredson H. Superior short-term results with eccentric calf muscle training compared to concentric training in a randomized prospective multicenter study on patients with chronic Achilles tendinosis. Knee Surg Sports Traumatol Arthrosc 2001;9:42-7.

25 Yu J, Park D, Lee G. Effect of eccentric strengthening on pain, muscle strength, endurance, and functional fitness factors in male patients with Achilles tendinopathy. Am J Phys Med Rehabil 2013;92:68-76.

26 Stevens M, Tan C-W. Effectiveness of the Alfredson protocol compared with a lower repetition-volume protocol for midportion Achilles tendinopathy: a randomized controlled trial. J Orthop Sports Phys Ther 2014;44:59-67.

27 da CRA, Dias AN, Santos MB, et al. Comparative study of two protocols of eccentric exercise on knee pain and function in athletes with Patellar tendinopathy: randomized controlled study. Revista Brasileira de Medicina do Esporte 2012;18:167-70.

28 Moher D, Liberati A, Tetzlaff J, et al. Preferred reporting items for systematic reviews and meta-analyses: the PRISMA statement. $J$ Clin Epidemiol 2009;62:1006-12.

29 Escriche-Escuder A, Casaña J, Cuesta-Vargas Al. Progression criteria in loading exercise programmes in lower limb tendinopathy: a protocol for a systematic review and meta-analysis. BMJ Open 2019;9:e032940.

30 Ferguson B. ACSM's Guidelines for Exercise Testing and Prescription. J Can Chiropr Assoc 2014;58:328.

31 Maher CG, Sherrington C, Herbert RD, et al. Reliability of the PEDro scale for rating quality of randomized controlled trials. Phys Ther 2003;83:713-21.

32 de Morton NA. The PEDro scale is a valid measure of the methodological quality of clinical trials: a demographic study. Aust $J$ Physiother 2009;55:129-33.

33 Cohen J. Statistical power analysis for the behavioral sciences. Hillsdale, NJ: Erlbaum Associates, 1988.

34 van Ark M, Cook JL, Docking SI, et al. Do isometric and isotonic exercise programs reduce pain in athletes with Patellar tendinopathy in-season? a randomised clinical trial. J Sci Med Sport 2016;19:702-6.

35 Ganderton C, Semciw A, Cook J, et al. Gluteal loading versus sham exercises to improve pain and dysfunction in postmenopausal women with greater trochanteric pain syndrome: a randomized controlled trial. J Womens Health 2018;27:815-29.

36 Clifford C, Paul L, Syme G, et al. Isometric versus isotonic exercise for greater trochanteric pain syndrome: a randomised controlled pilot study. BMJ Open Sport Exerc Med 2019;5:e000558.

37 Gatz M, Betsch M, Dirrichs T, et al. Eccentric and isometric exercises in Achilles tendinopathy evaluated by the VISA-A score and shear wave elastography. Sports Health 2020;12:373-81.

38 Niesen-Vertommen SL, Taunton JE, Clement DB, et al. The effect of eccentric versus concentric exercise in the management of Achilles tendonitis. Clin J Sport Med 1992;2:109-13.

39 Jonsson $\mathrm{P}$, Alfredson $\mathrm{H}$. Superior results with eccentric compared to concentric quadriceps training in patients with jumper's knee: a prospective randomised study. Br J Sports Med 2005;39:847-50.

40 Rompe JD, Nafe B, Furia JP, et al. Eccentric loading, shock-wave treatment, or a wait-and-see policy for tendinopathy of the main body of tendo Achillis: a randomized controlled trial. Am J Sports Med 2007;35:374-83.

41 Petersen W, Welp R, Rosenbaum D. Chronic Achilles tendinopathy: a prospective randomized study comparing the therapeutic effect of eccentric training, the AirHeel brace, and a combination of both. Am J Sports Med 2007;35:1659-67. 
42 Stefansson $\mathrm{SH}$, Brandsson S, Langberg $\mathrm{H}$, et al. Using pressure massage for Achilles tendinopathy: a single-blind, randomized controlled trial comparing a novel treatment versus an eccentric exercise protocol. Orthop J Sports Med 2019;7:232596711983428.

43 Kearney RS, Parsons N, Costa ML. Achilles tendinopathy management: a pilot randomised controlled trial comparing plateletrichplasma injection with an eccentric loading programme. Bone Joint Res 2013;2:227-32.

44 Bahr R, Fossan B, Løken S, et al. Surgical treatment compared with eccentric training for Patellar tendinopathy (jumper's knee). A randomized, controlled trial. J Bone Joint Surg Am 2006;88:1689-98.

45 Visnes $\mathrm{H}$, Hoksrud A, Cook J, et al. No effect of eccentric training on jumper's knee in volleyball players during the competitive season: a randomized clinical trial. Clin J Sport Med 2005;15:227-34.

46 Young MA, Cook JL, Purdam CR, et al. Eccentric decline squat protocol offers superior results at 12 months compared with traditional eccentric protocol for Patellar tendinopathy in volleyball players. Br J Sports Med 2005;39:102-5.

47 Horstmann T, Jud HM, Fröhlich V, et al. Whole-Body vibration versus eccentric training or a wait-and-see approach for chronic Achilles tendinopathy: a randomized clinical trial. J Orthop Sports Phys Ther 2013;43:794-803.

48 Yelland MJ, Sweeting KR, Lyftogt JA, et al. Prolotherapy injections and eccentric loading exercises for painful Achilles tendinosis: a randomised trial. Br J Sports Med 2011;45:421-8.

49 Zhang B-meng, Zhong L-wei, Xu S-wei, et al. Acupuncture for chronic Achilles tendnopathy: a randomized controlled study. Chin $J$ Integr Med 2013;19:900-4.

50 Frohm A, Halvorsen K, Thorstensson A. Patellar tendon load in different types of eccentric squats. Clin Biomech 2007;22:704-11.

51 Chester R, Costa ML, Shepstone L, et al. Eccentric calf muscle training compared with therapeutic ultrasound for chronic Achilles tendon pain--a pilot study. Man Ther 2008;13:484-91.

52 Nørregaard J, Larsen CC, Bieler T, et al. Eccentric exercise in treatment of Achilles tendinopathy. Scand J Med Sci Sports 2007;17:133-8.

53 Jensen K, Di Fabio RP. Evaluation of eccentric exercise in treatment of Patellar tendinitis. Phys Ther 1989;69:211-6.

54 Roos EM, Engström M, Lagerquist A, et al. Clinical improvement after 6 weeks of eccentric exercise in patients with mid-portion Achilles tendinopathy -- a randomized trial with 1-year follow-up. Scand J Med Sci Sports 2004:14:286-95.

55 Cannell LJ, Taunton JE, Clement DB, et al. A randomised clinical trial of the efficacy of drop squats or leg extension/leg curl exercises to treat clinically diagnosed jumper's knee in athletes: pilot study. $\mathrm{Br} \mathrm{J}$ Sports Med 2001;35:60-4.
56 Alfredson H, Ohberg L, Forsgren S. Is vasculo-neural ingrowth the cause of pain in chronic Achilles tendinosis? an investigation using ultrasonography and colour Doppler, immunohistochemistry, and diagnostic injections. Knee Surg Sports Traumatol Arthrosc 2003;11:334-8.

57 Farrar JT, Young JP, LaMoreaux L, et al. Clinical importance of changes in chronic pain intensity measured on an 11-point numerical pain rating scale. Pain 2001;94:149-58.

58 Jakobsen TL, Kehlet H, Husted H, et al. Early progressive strength training to enhance recovery after fast-track total knee arthroplasty: a randomized controlled trial. Arthritis Care Res 2014;66:1856-66.

59 Riel H, Jensen MB, Olesen JL, et al. Self-dosed and pre-determined progressive heavy-slow resistance training have similar effects in people with plantar fasciopathy: a randomised trial. J Physiother 2019:65:144-51.

60 Saithna A, Gogna R, Baraza N, et al. Eccentric exercise protocols for patella tendinopathy: should we really be withdrawing athletes from sport? A systematic review. Open Orthop J 2012;6:553-7.

61 Rompe JD, Furia J, Maffulli N. Eccentric loading versus eccentric loading plus shock-wave treatment for midportion Achilles tendinopathy: a randomized controlled trial. Am J Sports Med 2009;37:463-70.

62 Herrington L, McCulloch R. The role of eccentric training in the management of Achilles tendinopathy: a pilot study. Physical Therapy in Sport 2007;8:191-6.

63 Tumilty S, Mani R, Baxter GD. Photobiomodulation and eccentric exercise for Achilles tendinopathy: a randomized controlled trial. Lasers Med Sci 2016;31:127-35.

64 Tsehaie J, Poot DHJ, Oei EHG, et al. Value of quantitative MR parameters in predicting and evaluating clinical outcome in conservatively treated patients with chronic midportion Achilles tendinopathy: a prospective study. J Sci Med Sport 2017;20:633-7.

65 Shalabi A, Kristoffersen-Wilberg M, Svensson L, et al. Eccentric training of the gastrocnemius-soleus complex in chronic Achilles tendinopathy results in decreased tendon volume and intratendinous signal as evaluated by MRI. Am J Sports Med 2004;32:1286-96.

66 Gärdin A, Movin T, Svensson L, et al. The long-term clinical and MR results following eccentric calf muscle training in chronic Achilles tendinosis. Skeletal Radiol 2010;39:435-42.

67 Hasani F, Haines TP, Munteanu SE, et al. Efficacy of different load intensity and time-under-tension calf loading protocols for Achilles tendinopathy (the LOADIT trial): protocol for a randomised pilot study. Pilot Feasibility Stud 2020;6:99.

68 Morrissey D, Roskilly A, Twycross-Lewis R, et al. The effect of eccentric and concentric calf muscle training on Achilles tendon stiffness. Clin Rehabil 2011;25:238-47. 